University of Nebraska - Lincoln

DigitalCommons@University of Nebraska - Lincoln

Roger Kirby Publications

Research Papers in Physics and Astronomy

May 1984

\title{
Effect of Nickel Impurities on Charge-density-wave Formation in $\mathrm{TaS}_{3}$
}

\author{
M.H. Rashid \\ University of Nebraska - Lincoln \\ David J. Sellmyer \\ University of Nebraska-Lincoln, dsellmyer@unl.edu \\ Roger D. Kirby \\ University of Nebraska-Lincoln, rkirby1@unl.edu
}

Follow this and additional works at: https://digitalcommons.unl.edu/physics_kirby

Part of the Physics Commons

Rashid, M.H.; Sellmyer, David J.; and Kirby, Roger D., "Effect of Nickel Impurities on Charge-density-wave Formation in $\mathrm{TaS}_{3}$ " (1984). Roger Kirby Publications. 25.

https://digitalcommons.unl.edu/physics_kirby/25

This Article is brought to you for free and open access by the Research Papers in Physics and Astronomy at DigitalCommons@University of Nebraska - Lincoln. It has been accepted for inclusion in Roger Kirby Publications by an authorized administrator of DigitalCommons@University of Nebraska - Lincoln. 


\title{
Effect of nickel impurities on charge-density-wave formation in $\mathrm{TaS}_{3}$
}

\author{
M. H. Rashid, D. J. Sellmyer, and R. D. Kirby \\ Behlen Laboratory of Physics, University of Nebraska, Lincoln, Nebraska 68588
}

(Received 13 October 1983)

\begin{abstract}
Introduction of nickel impurities into $\mathrm{TaS}_{3}$ has the effect of smearing the charge-density-wave transitions in $\mathrm{TaS}_{3}$ of both orthorhombic and monoclinic phases. The orthorhombic doped crystals show non-Ohmic conductivity beyond threshold fields that are much larger than the threshold field of the pure phase, possibly due to additional pinning of the charge-density wave by nickel impurities. The charge-density-wave state is completely destroyed as 10 at. \% or more Ta atoms are replaced by $\mathrm{Ni}$ atoms and metallic behavior between 4.2 and $300 \mathrm{~K}$ is observed in these cases.
\end{abstract}

\section{INTRODUCTION}

The quasi-one-dimensional fibrous compound $\mathrm{TaS}_{3}$ exists in two phases-orthorhombic ${ }^{1}$ and monoclinic. ${ }^{2}$ The orthorhombic phase has a large unit cell containing $24 \mathrm{Ta}$ chains and having the following dimensions: $a=36.804$ $\AA, b=15.173 \AA$, and $c=3.340 \AA$. The space-group symmetry is $C 222_{1}$. This phase shows a commensurate charge-density-wave (CDW) transition at $215 \mathrm{~K}^{3-5} \mathrm{Re}-$ cently observations of nonlinear conductivity, ${ }^{6,7}$ frequency-dependent conductivity, ${ }^{8}$ and narrow-band noise ${ }^{9}$ in orthorhombic $\mathrm{TaS}_{3}$ have been reported. The observed non-Ohmic conductivity has been interpreted in terms of depinning of the CDW's pinned by the lattice, impurities or dislocations at electric fields higher than a threshold field.

Monoclinic $\mathrm{TaS}_{3}$, on the other hand, is isotypic with $\mathrm{NbSe}_{3}$ and $\mathrm{TaSe}_{3}$ (space group $P 2_{1} / m$ ) with the following unit-cell dimensions: ${ }^{2} \quad a=9.515 \AA$ А $b=3.3412 \AA$, $c=14.912 \AA$, and $\beta=109.99^{\circ}$. This phase exhibits two independent incommensurate CDW transitions at $T_{1}=240$ $\mathrm{K}$ and $T_{2}=160 \mathrm{~K} .^{10}$ Hasegawa et al. ${ }^{11}$ have observed nonlinear conductivity and broad-band noise in monoclinic $\mathrm{TaS}_{3}$.

Attempts have been made to theoretically understand the effect of impurities on a CDW state. ${ }^{12,13}$ In this paper we report a systematic experimental study of the effect of nickel impurities on the CDW condensate in $\mathrm{TaS}_{3}$.

\section{EXPERIMENTAL METHODS}

Crystals of the form $\mathrm{Ni}_{x} \mathrm{Ta}_{1-x} \mathrm{~S}_{3}$ are grown by the vapor-phase transport method from fine powders of constituent elements. The starting material is sealed under vacuum in quartz tubes and heated at $685^{\circ} \mathrm{C}$ in a furnace for three weeks and then slow-cooled to room temperature in a week. Fibrous crystals of maximum dimensions $10 \times 0.03 \times 0.01 \mathrm{~mm}^{3}$ are obtained by this method. There is no apparent distinction in physical appearance between the nickel-doped and the pure $\mathrm{TaS}_{3}$ crystals.

Stoichiometries of the crystals are determined by the $\mathrm{x}$ ray fluorescence method. However, this task has been complicated in the case of nickel-doped crystals by the fact that crystals from the same growth tube have some- what different nickel contents as could be judged from their distinctive resistivity behavior. In addition, the fibrous crystals are extremely thin $(\sim 0.01 \mathrm{~mm})$ so fluorescence measurements are not possible on individual crystals. For small Ni concentrations, $x \leq 0.08$, it was necessary to determine $x$ in an average way by fluorescence measurements on a number of their crystals. Thus only very approximate $x$ values are known for these crystals. It is estimated that the $\mathrm{Ni}$ concentrations of the two "dilute" crystals are given by $x=0.04 \pm 0.02, x=0.08 \pm 0.02$. The stoichiometry problem is much less severe in the case of $\mathrm{Ni}_{0.1} \mathrm{Ta}_{0.9} \mathrm{~S}_{3}$ and $\mathrm{Ni}_{0.13} \mathrm{Ta}_{0.87} \mathrm{~S}_{3}$ which show metallic behavior. Crystals from the same growth tube in these cases show similar resistivity behavior. In these cases we estimate the uncertainty in $x$ to be about \pm 0.01 .

The dc resistivity is measured along the chain axis by the four-probe method. Silver paint is used to fabricate the current and voltage contacts. The sample is mounted on a "dip probe" which is lowered slowly in a liquidhelium storage Dewar to measure the sample resistance as a function of temperature down to $4.2 \mathrm{~K}$. Dimensions of the sample are measured by a traveling microscope.

\section{RESULTS AND DISCUSSION}

Figure 1 shows the temperature dependence of the normalized low-field resistivity of pure and nickel-doped $\mathrm{TaS}_{3}$ samples plotted versus $1000 / T$ in the temperature range $55-300 \mathrm{~K}$. Curve $a$ of Fig. 1 gives the resistivity behavior of pure orthorhombic $\mathrm{TaS}_{3}$. The resistivity increases monotonically with decreasing temperature below the CDW transition temperature of $220 \mathrm{~K}$. The slope of the curve is continually changing with temperature, so that the resistivity does not follow a simple expression of the form $\rho=\rho_{0} \exp \left(\Delta / 2 k_{B} T\right)$. This is consistent with the previously observed resistivity behavior. ${ }^{6,10,14}$ The roomtemperature resistivity value is $2 \times 10^{-4} \Omega \mathrm{cm}$. The resistivity approaches a saturation value of $\sim 10^{2} \Omega \mathrm{cm}$ at 4.2 $\mathrm{K}$. We have not observed any monoclinic $\mathrm{TaS}_{3}$ crystal mixed with the pure orthorhombic phase in our growth tube as has been observed by Roucau et al. ${ }^{10}$ (their growth temperature is $35^{\circ} \mathrm{C}$ lower than ours). However, both orthorhombic and monoclinic varieties are present in the same growth tube in the case of nickel-doped crystals. 


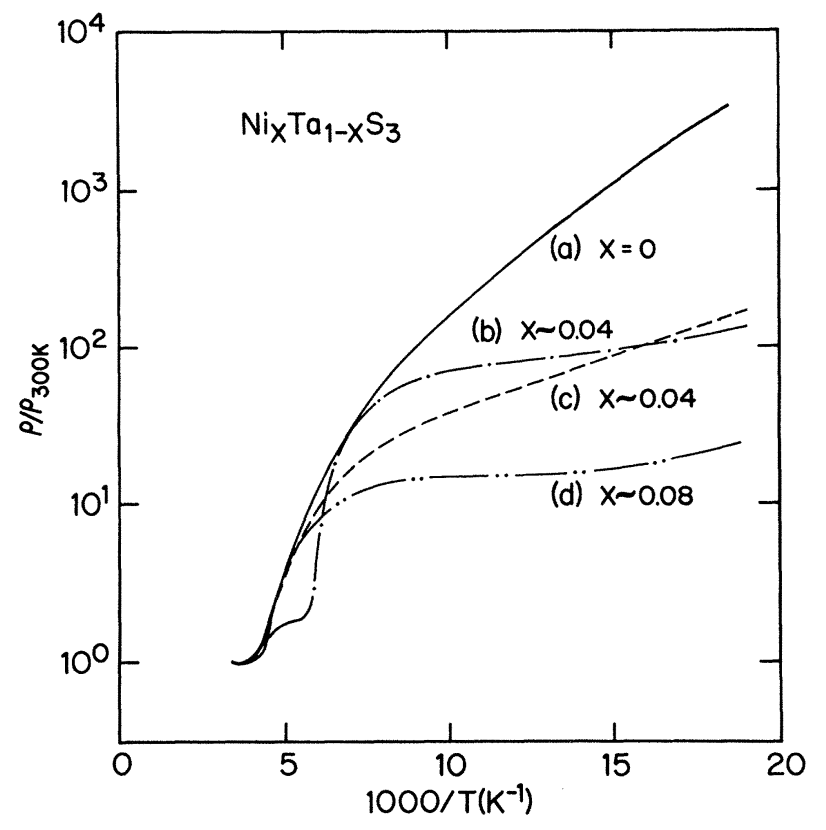

FIG. 1. Resistivity behavior of orthorhombic $\mathrm{TaS}_{3}(a)$, monoclinic $\mathrm{Ni}_{\sim 0.04} \mathrm{Ta}_{0.96} \mathrm{~S}_{3}(b)$, orthorhombic $\mathrm{Ni}_{\sim 0.04} \mathrm{Ta}_{0.96} \mathrm{~S}_{3}$ (c), and orthorhombic $\mathrm{Ni}_{\sim 0.08} \mathrm{Ta}_{0.92} \mathrm{~S}_{3}(d)$.

Identification of the correct phase, in this case, is done by the temperature dependence of the resistivity. There is only one CDW transition at $220 \mathrm{~K}$ in orthorhombic crystals and the resistivity varies smoothly with temperature below this transition temperature. On the other hand, there are two distinct CDW transitions at $T_{1}=240 \mathrm{~K}$ and $T_{2}=160 \mathrm{~K}$ in monoclinic crystals. A sample of the orthorhombic phase can be ascertained by the absence of any resistivity anomaly at $160 \mathrm{~K}$. In Fig. 1 curves $c$ and $d$ show the plots of the normalized resistivity versus $1 / T$ for $\mathrm{Ni}_{\sim 0.04} \mathrm{Ta}_{0.96} \mathrm{~S}_{3}$ and $\mathrm{Ni}_{\sim 0.08} \mathrm{Ta}_{0.92} \mathrm{~S}_{3}$, respectively, of the orthorhombic phase. Although it is not obvious from Fig. 1, the CDW transition temperatures of these nickeldoped orthorhombic crystals are slightly higher $\left(10-15^{\circ} \mathrm{C}\right)$ than the pure crystal. But a more interesting effect of the nickel impurity is the systematic lowering of the slopes of the curves with increasing nickel content. This would mean that a tendency toward metallic behavior is being induced by the nickel impurities. This suggests that the long-range coherence of the CDW state is being partially destroyed by the impurities. A similar effect is also observed in monoclinic $\mathrm{Ni}_{\sim 0.04} \mathrm{Ta}_{0.96} \mathrm{~S}_{3}$ as shown in curve $b$ of Fig. 1. In this case, however, there is no noticeable change from the pure crystal in the two transition temperatures. Only the slope of the curve is lower than in the pure monoclinic phase. ${ }^{10,11}$ With further increase of nickel impurities, the coherence of the CDW state is completely destroyed and the doped crystals ultimately become metallic. Figure 2 shows this metallic behavior for $\mathrm{Ni}_{0.1} \mathrm{Ta}_{0.9} \mathrm{~S}_{3}$. Qualitatively similar behavior is observed for $\mathrm{Ni}_{0.13} \mathrm{Ta}_{0.87} \mathrm{~S}_{3}$. Identification of the actual phases of individual metallic crystals is not possible from the resistivity behavior alone since the resistivity anomalies of the CDW state are now absent. Both phases, orthorhombic and monoclinic, a mixture of the two, or a

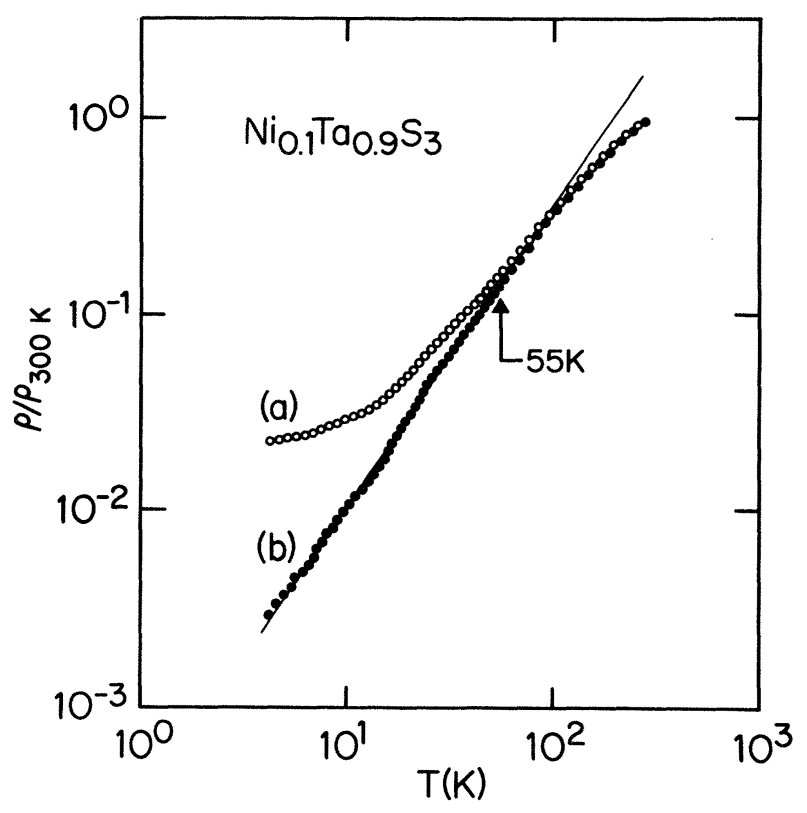

FIG. 2. Temperature dependence of the resistivity of $\mathrm{Ni}_{0.1} \mathrm{Ta}_{0.9} \mathrm{~S}_{3}$ as measured $(a)$ and with the residual resistivity subtracted $(b)$.

new phase may be present among these metallic crystals. Given the complex structures of pure $\mathrm{TaS}_{3}$ with many atoms per unit cell and the fact that we were able to obtain only very small quantities of these crystals, it was not possible to determine the unit-cell parameters of the metallic crystals by $\mathrm{x}$-ray measurements. The absolute resistivity values of these crystals at room temperature are comparable to those of pure $\mathrm{TaS}_{3}$ within our accuracy of measurements which is, of course, not very good due to difficulties in measuring the dimensions of these thin crystals. The resistivity ratio, $\rho_{300} / \rho_{4.2 \mathrm{~K}}$, is $\sim 50$ for $\mathrm{Ni}_{0.1} \mathrm{Ta}_{0.9} \mathrm{~S}_{3}$ and $\sim 20$ for $\mathrm{Ni}_{0.13} \mathrm{Ta}_{0.87} \mathrm{~S}_{3}$. These relatively high ratios suggest that there may well be considerable order in the Ni atomic positions; that is, the Ni atoms may not enter the lattice in a random fashion. The curve $a$ of Fig. 2 shows the temperature dependence of the normalized resistivity and the curve $b$ is obtained after subtracting the residual resistivity which has been estimated by extrapolating the curve $a$ to $T=0 \mathrm{~K}$. The linear behavior of the curve $b$ in the low-temperature region suggests that the resistivity of the metallic crystals can be expressed as $\rho(T)=\rho_{0}+C T^{n}$, where $\rho_{0}$ is the temperature-independent residual resistivity. The exponent has a value $n=1.5 \pm 0.1$ for $\mathrm{Ni}_{0.1} \mathrm{Ta}_{0.9} \mathrm{~S}_{3}$ below $55 \mathrm{~K}$ and a value $n=1.3 \pm 0.1$ for $\mathrm{Ni}_{0.13} \mathrm{Ta}_{0.87} \mathrm{~S}_{3}$ below $90 \mathrm{~K}$. In both cases the resistivity behavior goes approximately as $T^{0.8}$ at room temperature and the tendency to saturation would likely be seen at a higher temperature. Such saturation effects are not unexpected in metals with resistivity values above about 200 $\mu \Omega \mathrm{m} .{ }^{15}$

The normalized dc conductivity, measured as a function of dc electric field across the length of the sample, is shown in Fig. 3 for orthorhombic $\mathrm{TaS}_{3}$ at $T=130 \mathrm{~K}$. The conductivity is Ohmic for electric fields less than $\sim 1.5 \mathrm{~V} / \mathrm{cm}$. Above this threshold field, the normalized 


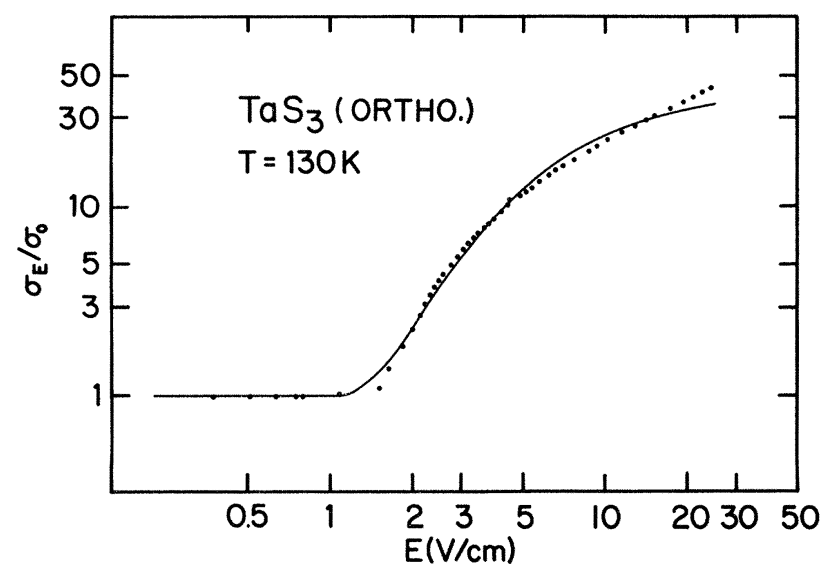

FIG. 3. Electric field dependence of the conductivity of orthorhombic $\mathrm{TaS}_{3}$ at $130 \mathrm{~K}$. The curve is a fit to Eq. (1) with the parameters given in the text.

conductivity rises steadily up to a factor of 60 for an electric field of $35 \mathrm{~V} / \mathrm{cm}$. At electric fields higher than 35 $\mathrm{V} / \mathrm{cm}$, there is a steady drift in the value of the sample resistance, indicating sample heating. Our observed field dependence of conductivity of $\mathrm{TaS}_{3}$ is consistent with the observation of nonlinear conductivity by Thompson, Zettle, and Grüner. ${ }^{6}$ These authors have compared their nonlinear conductivity results to the Zener-tunneling model of Bardeen ${ }^{16}$ and also to the classical model of Grüner et $a l .{ }^{17}$ The classical model is inadequate because it predicts that the differential conductivity $(d I / d V)$ diverges at the threshold field, a result not seen experimentally. It was found ${ }^{17}$ that the data could be fitted reasonably well by the Bardeen expression for quantummechanical tunneling,

$$
\sigma(E)=A\left(1-E_{T} / E\right) \exp \left(-E_{0} / E\right),
$$

where $E_{T}$ and $E_{0}$ are threshold and single-particle Zener fields, respectively, and $A$ is a constant. Figure 3 also shows a fit to Eq. (1) which was obtained with $E_{T}=1.1$ $\mathrm{V} / \mathrm{cm}, E_{0}=5.5 \mathrm{~V} / \mathrm{cm}$, and $A=44$. These parameters are similar to those obtained by Zettl et al. ${ }^{6}$ However, it is seen that there are some quantitative discrepancies between Eq. (1) and experiment, particularly at high fields where sample heating may not be negligible. Of most interest for the present work is the behavior of the nonOhmic conductivity in the Ni-doped samples. Figures 4 and 5 show this dependence for both orthorhombic and monoclinic $\mathrm{Ni}_{\sim 0.04} \mathrm{Ta}_{0.96} \mathrm{~S}_{3}$ at $130 \mathrm{~K}$. Also shown are the best fits we could obtain to Eq. (1). The parameters for the orthorhombic and monoclinic crystals are $E_{T}=15$ $\mathrm{V} / \mathrm{cm}, E_{0}=75 \mathrm{~V} / \mathrm{cm}$, and $A=20$, and $E_{T}=1.3 \mathrm{~V} / \mathrm{cm}$, $E_{0}=6.5 \mathrm{~V} / \mathrm{cm}$, and $A=13$, respectively. For the orthorhombic case the fit is particularly poor, although it is clear that the threshold fit has gone up by a large factor $(\sim 14)$. The sharp threshold field appears to be lost in this case, thus suggesting inhomogeneities in the electric field caused by the impurities and perhaps a loss of coherence of the CDW. On the other hand, the monoclinic structure with the same nominal Ni concentration shows a threshold field much smaller than that of the orthorhombic phase. These results suggest that the specific struc-

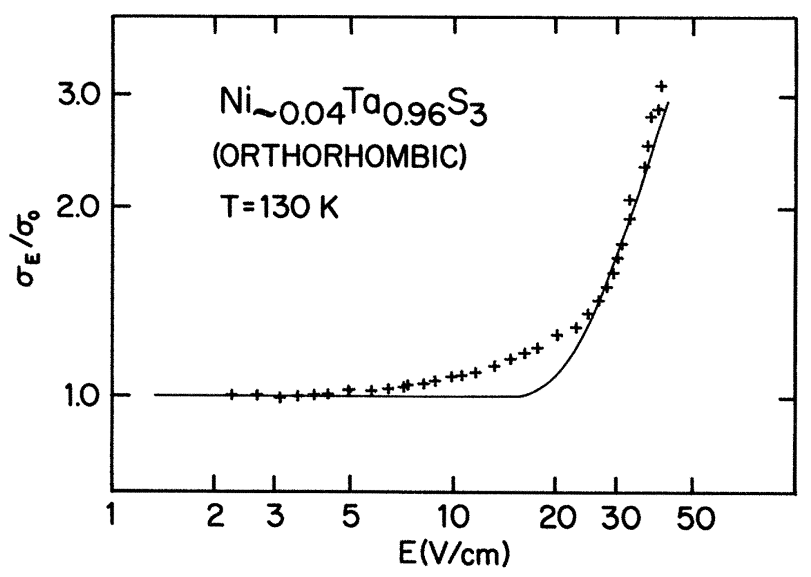

FIG. 4. Electric field dependence of the conductivity of orthorhombic $\mathrm{Ni}_{\sim 0.04} \mathrm{Ta}_{0.96} \mathrm{~S}_{3}$ at $130 \mathrm{~K}$. The curve is a fit to Eq. (1) with the parameters given in the text.

ture, whether the impurity atoms are substitutional or interstitial, plays an important role in the pinning of the CDW. A related point is that Hsieh et al. ${ }^{18}$ have found that doping orthorhombic $\mathrm{TaS}_{3}$ with $\mathrm{Nb}$ and Se depresses and broadens the Peierls transition. Of particular note is that these workers found at $130 \mathrm{~K} E_{T} \simeq 0.2 \mathrm{~V} / \mathrm{cm}$ for pure $\mathrm{TaS}_{3}$ (compared to our $1.1 \mathrm{~V} / \mathrm{cm}$ ) and that very small concentrations of isoelectronic impurities such as $\mathrm{Nb}$ or Se had a strong effect on $E_{T}$. For example, they found that replacing only 2 parts in 1000 of $\mathrm{Ta}$ by $\mathrm{Nb}$ changed $E_{T}$ from 0.2 to $15 \mathrm{~V} / \mathrm{cm}$. Thus, in comparison, the effect of impurities in our Ni-doped orthorhombic crystals seems relatively weak. One might speculate from this that the Ni impurities, which are not isoelectronic, do not go into the $\mathrm{TaS}_{3}$ structure as substitutions for Ta. This points toward a need for detailed structural information on the doped crystals, and also for an accurate method of measuring the impurity content of individual crystals in order to derive a quantitative relation between threshold field and impurity concentration. Such information is not easily obtainable for these crystals because of the small size of the doped crystals that can be grown.

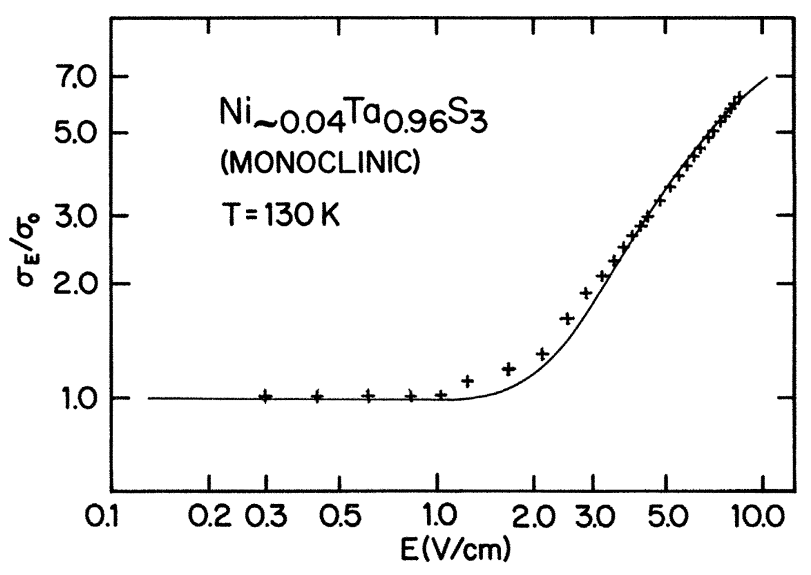

FIG. 5. Electric field dependence of the conductivity of monoclinic $\mathrm{Ni}_{\sim 0.04} \mathrm{Ta}_{0.96} \mathrm{~S}_{3}$ at $130 \mathrm{~K}$. The curve is a fit to Eq. (1) with the parameters given in the text. 
In summary, we have shown qualitatively that $\mathrm{Ni}$ impurities have the effect of increasing the threshold field for the depinning of CDW's. Rough agreement between the $\sigma(E)$ data and the Bardeen tunneling theory was obtained for nominally pure orthorhombic $\mathrm{TaS}_{3}$ and monoclinic Ni-doped $\mathrm{TaS}_{3}$. However, neither the classical model nor the tunneling model is capable of accounting for all of the experiments on $\mathrm{TaS}_{3}$ and $\mathrm{NbSe}_{3} .{ }^{17}$ Recently Tua and Zawadowski have reviewed other theoretical models and introduced a modified classical model to describe the nonlinear conductivity of CDW systems with strong impurity pinning. ${ }^{19}$ Their model predicts that the differential conductivity is nonsingular at the threshold field in agreement with the results presented here and in Refs. 6 and 18.

\section{ACKNOWLEDGMENT}

We are grateful to the National Science Foundation for financial support under Grant No. DMR-81-10520.
${ }^{1}$ E. Bjerkelund and A. Kjekshus, Z. Anorg. Allgem. Chem. $\underline{328}$, 235 (1964).

${ }^{2}$ A. Meerschaut, J. Rouxel, P. Haen, P. Monceau, and M. Nunez-Regueiro, J. Phys. (Paris) Lett. 40, L157 (1979).

${ }^{3}$ T. Sambongi, K. Tsutsumi, Y. Shiozaki, M. Yamamoto, K. Yamaya, and Y. Abe, Solid State Commun. 22, 729 (1977).

${ }^{4}$ M. Ido, K. Tsutsumi, T. Sambongi, and N. Mori, Solid State Commun. 29, 399 (1979).

${ }^{5} \mathrm{~K}$. Tsutsumi, T. Sambongi, S. Kagoshima, and T. Ishiguro, J. Phys. Soc. Jpn. 44, 1735 (1978).

${ }^{6}$ A. H. Thompson, A. Zettl, and G. Grüner, Phys. Rev. Lett. 47, 64 (1981); A. Zettl, G. Grüner, and A. H. Thompson, Phys. Rev. B 26, 5760 (1982).

${ }^{7}$ T. Takoshima, M. Ido, K. Tsutsumi, T. Sambongi, S. Honma, K. Yamaya, and Y. Abe, Solid State Commun. 35, 911 (1980).

${ }^{8}$ C. M. Jackson, A. Zettl, G. Grüner, and A. H. Thompson, Solid State Commun. 39, 531 (1981).

${ }^{9}$ G. Grüner, A. Zettl, W. G. Clark, and A. H. Thompson, Phys. Rev. B 23, 6813 (1981).
${ }^{10}$ C. Roucau, R. Ayroles, P. Monceau, L. Guemas, A. Meerschaut, and J. Rouxel, Phys. Status Solidi A 62, 483 (1980).

${ }^{11}$ K. Hasegawa, A. Maeda, S. Uchida, and S. Tanaka, Solid State Commun. 44, 881 (1982).

${ }^{12}$ L. J. Sham and B. R. Patton, Phys. Rev. B 13, 3151 (1976).

${ }^{13}$ R. A. Klemm and J. R. Schrieffer, Phys. Rev. Lett. 51, 47 (1983).

${ }^{14}$ E. Bjerkelund, J. H. Fermor, and A. Kjekshus, Acta Chem. Scand. 20, 1836 (1966).

${ }^{15} \mathrm{See}$, for example, P. J. Cote and L. V. Meisel, in Glassy Metals I, edited by H. J. Güntherodt and H. Beck (Springer, Berlin, 1981), p. 141.

16J. Bardeen, Phys. Rev. Lett. 45, 1978 (1980).

${ }^{17}$ See G. Grüner, Comments Solid State Phys. 10, 183 (1983), and references therein.

${ }^{18}$ Pei-Ling Hsieh, F. de Czito, A. Janossy, and G. Grüner, J. Phys. (Paris) Colloq. 44, C3-1753 (1984).

${ }^{19}$ P. F. Tua and A. Zawadowski, Solid State Commun. 49, 19 (1984). 